\title{
Phase Offset Between Slow Oscillatory Cortical Inputs Influences Competition in a Model of the Basal Ganglia
}

\author{
Zafeirios Fountas and Murray Shanahan
}

\begin{abstract}
Low-frequency oscillations have been the target of extensive research both in cortical structures and in the basal ganglia, due to numerous reports of associations with brain disorders and the normal functioning of the brain. Whereas a number of computational models of the basal ganglia investigate these phenomena, these models tend to focus on intrinsic oscillatory mechanisms, neglecting evidence that points to the cortex as the origin of this oscillatory behaviour. In this work we constructed a neural model of the basal ganglia circuitry and used it to investigate the relationship between frequency of oscillatory cortical input, dopamine and the effectiveness of the basal ganglia as an action selection device. Our simulations show the impact of the phase offset between different cortical inputs. This was found to be highly dependent on the frequency band and to have a strong influence on basal ganglia effectiveness. In addition, the level of dopamine in the system was found to modulate this effect, also depending on the input's frequency band.
\end{abstract}

\section{INTRODUCTION}

$\mathbf{T}$ HE BASAL GANGLIA (BG) are a group of subcortical nuclei that show remarkable similarities, both anatomically and functionally, across vertebrate nervous system. Both their physical location and their broad bidirectional connectivity with major cortical areas, the limbic system and the thalamus, place the BG in a key position to modulate the information flow between the cortex and the body. In addition, their strictly topographic organization on different scales suggests that through the BG, common modulatory operations are applied to functionally different channels of information flow.

The above features have led to the widely held hypothesis that the BG constitute a critical component for the action selection system of the vertebrate brain [1]. Since then, a substantial number of models have been built on this foundation including abstract mathematical models [2], [3] and spiking neuron models [4], [5], [6], [7] and used to control either physically embodied [8] or simulated [9] robotic agents.

Another major feature of the function of the $\mathrm{BG}$ is the existence of strong oscillatory activity in a wide spectrum of bands [10], [11]. Although a number of models in the literature have investigated this phenomenon before [4], [7], these models tend only to focus on mechanisms for generating oscillations intrinsically. One area that has not been examined so far is how oscillatory activity that is generated in higher cognitive areas can be dealt with the BG circuitry. Acquiring a better understanding of this behaviour could give more insight into the nature of this cortical input, the role

Zafeirios Fountas and Murray Shanahan are both with the Department of Computing, Imperial College London, London, United Kingdom, email: \{zfountas, m.shanahan\}@imperial.ac.uk of the $\mathrm{BG}$ in cognition and their relation to a number of disorders such as Parkinson's disease (PD), a severe brain disorder caused by dopamine depletion in the BG.

In this study we address this issue by means of a biologically plausible neural model of BG circuitry. Using this model, we carried out an analysis of the relationship between frequency and the effectiveness of the BG as an action selection device.

We found that in low frequencies $(<30 \mathrm{~Hz})$, the phase offset of coherent cortical signals with different amplitudes can have a strong influence on BG selectivity, while in the gamma band, this effect disappears. In particular, coherent high-beta $(20-30 \mathrm{~Hz})$ oscillating input signals were found to favour the effectiveness of the BG model significantly, but only in the case that the phase of a strong input signal precedes in time the phase of a second, weaker signal, with a small offset around $\frac{\pi}{2}$. On the other hand, hippocampal theta $(4-10 \mathrm{~Hz})$ and low-beta $(13-20 \mathrm{~Hz})$ oscillations had a negative influence on BG selectivity, especially in the case of a phase offset greater than $\pi$. Finally, we investigated the effect of dopamine on the process of selection which was found to modulate the frequency spectrum of the previous effects.

This paper is organized as follows. Section II overviews the anatomy and function of the BG, with particular attention to their relation with neural oscillations and Parkinson's disease. Section III describes our experimental approach. The high-level architecture of the BG model is presented, the low-level neuron model is defined, various parameter choices are justified, and the experimental procedure is described. Section IV presents the results of these experiments, and Section V links our results with findings from the recent literature. Section VI offers some concluding remarks and directions for future work.

\section{BACKGROUND}

\section{A. Anatomy}

The BG internal structure comprises a canonical circuit, replicated in parallel and in different scales. On the highest level, this circuit is part of a set of parallel loops involving distinct areas of the cortex such as the motor or associative areas, the BG and the thalamus [12]. Moreover, these macroscopic loops can be further divided two more times, into microscopic sub-loops (or channels) that also run in parallel.

As mentioned in the introduction, this topographic organization implies that the BG act as a fundamental processing unit with common operations applied to functionally different cortical areas. It has been further suggested that in the 
lowest scale, each parallel microscopic channel represents a competing piece of information or an action [1], [2], [4].

Anatomically, the biological BG consist of two input nuclei, the subthalamic nucleus (STN) and the striatum, two output nuclei, the substantia nigra pars reticulata $(\mathrm{SNr})$ and the internal globus pallidus (GPi) as well as the external globus pallidus (GPe) and finally the substantia nigra pars compacta ( $\mathrm{SNc}$ ), a group of dopaminergic neurons that modulate both internal and external structures. Dopamine has a strong effect on the largest component of the $\mathrm{BG}$, the striatum, and it is considered very important for its operation.

The striatum comprises up to $97 \%$ medium spiny projection neurons (MSNs) and around $1 \%$ fast spiking interneurons (FSIs) interconnected by both chemical synapses and gap junctions [14], [15]. It can be further broken down into two populations of projection neurons based on the dominant type of their dopamine receptors that belong either to the D1or D2-like families. D1 striatal neurons are enhanced by the presence of dopamine while the latter has negative effect to D2 neurons (table II).

Cortical signals are initially projected to the striatum and STN, processed by the internal system and later transmitted to the thalamus via the inhibition of the SNr and GPi. Internally, although the structures are wired in a more complicated manner [13], two major pathways are thought to characterize the behaviour of the BG, corresponding to two distinct major thalamo-cortical loops [16], [2]. In the "direct" (or "selection") pathway, striatum D1 and STN neurons project their signals directly to the BG output nuclei $\mathrm{SNr}$ and GPi which in turn inhibit the thalamus and brainstem. STN neurons also take part in the second "indirect" (or "control") pathway and along with stratal D2 and GPe neurons modulate the effect of the direct pathway to the SNr-GPi complex.

\section{B. Oscillations and PD}

A major feature of the BG nuclei is their rich oscillatory activity which varies, depending on the health and the current cognitive state of the subject and is known to modulate their behaviour. This topic has attracted numerous studies that mainly involve patients of PD [17], [18], [19], [20], [21]. One important reason is that deep brain stimulation (DBS), a common surgical treatment for PD, provides researchers with the opportunity to record the spiking activity of multiple BG structures in human patients simultaneously.

Under Parkinsonian conditions, oscillations in the BG exhibit an excessive synchronization in the beta frequency band $(15-30 \mathrm{~Hz})$, as first indentified by [17], which is attenuated by treatment with anti-Parkinsonian medication [18]. In addition, slow oscillatory activity is linked to a number of particular symptoms, such as the inability of patients to initiate and execute movements [20] and resting tremor generation [19]. Although DBS is proven to relieve these symptoms and restore normal function, its underlying mechanism is still an active research area [21].

In healthy brains, though less studied, oscillatory phenomena are also present in the $\mathrm{BG}$, and cover a wider range of frequencies. Striatal local field potential recordings of rats during a radial maze task have revealed strong oscillatory activity at theta $(\sim 8 \mathrm{~Hz})$, beta $(\sim 20 \mathrm{~Hz})$, and gamma ( $\sim 50 \mathrm{~Hz})$ bands [11] with gamma oscillations (mainly at $40-80 \mathrm{~Hz}$ ) prevailing in the BG nuclei [22].

While the importance of low oscillations (such as beta) in the BG operation is beyond doubt, the literature is still lacking a widely accepted theory of their functional role and why they are less dominant under healthy conditions [23], [24]. Two hypotheses state that beta-band activity is responsible for the maintenance of the current sensorimotor or cognitive state [23] as well as that it encodes the likelihood of a new goal-oriented movement [25].

Computational models of the BG have been employed to identify the control mechanisms of oscillations, including [4], where oscillatory behaviour is associated with the action selection hypothesis, [3] where it is investigated whether beta oscillations can emerge from the interaction between STN and GPe and [5] where it is suggested that inhibition to GPe, caused by the striatum, is responsible to control oscillatory behaviour.

Although the above studies focus on oscillations within the BG, it has been argued that this activity is more likely to be generated externally, as a part of the thalamo-cortical loop [26], [27], [25] and maintain its coherence throughout the $\mathrm{BG}$.

In [28], low-frequency coherent oscillations were found to play a significant role in the communication between the rat prefrontal cortex (PFC) and hippocampus, two major inputs of the BG. Indeed, in this study, periods of theta coherence peaked when the rats were at a choice point on a $\mathrm{Y}$ maze, allowing new assemblies in the PFC to be formed. Also, in an earlier study [11], coherence was found to be strong between striatal $\sim 8 \mathrm{~Hz}$ oscillations and hippocampal theta, during task performance. These findings are in line with the view that cortical low-frequency oscillations not only influence the behaviour of the BG but they also play an important role in the process of selecting and performing an action. Our model vindicates this view, while it also provides a functional distinction between the theta, beta and gamma frequency bands, at the level of the BG.

\section{Methodology}

\section{A. Model architecture}

Our model comprises five distinct neural populations that account for the four major nuclei of the biological BG, shown in Figure 1. The internal connectivity is largely adopted from a well-established model by Humphries et al [4] and shapes the canonical circuitry that is described in section I.

Inside each nucleus, three largely isolated subgroups with an equal number of neurons correspond to three microscopic channels of the same microcircuit.

Connections between the striatum, GPe, GPi/SNr and afferent connections to STN are restricted to the same preand post-synaptic channel (topographic connections), while STN projections as well as recurrent connections within the GPe and the GPiSNr are equally distributed across all 


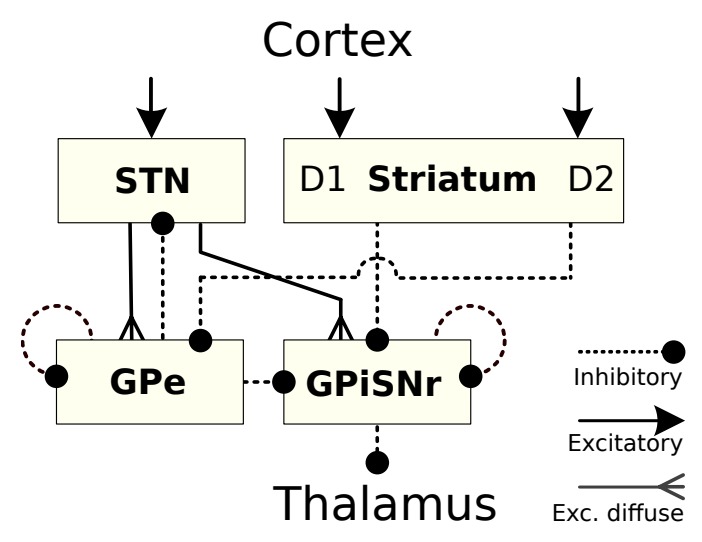

Fig. 1. The architecture of the basal ganglia model.

three channels. Each topographically connected pre-synaptic neuron has probability $p_{\text {con }}$ to be connected to a neuron in the post-synaptic group and for diffuse connections this probability is $p_{\text {con }} / N$, where $N=3$ is the number of channels. All synaptic parameters including connection probabilities, weights and delays are given in table III.

The estimated absolute number of neurons of the BG nuclei varies from a vast number of spiny projection neurons in the neostriatum to the relatively small GPi [29]. However, the inter-nuclei connectivity is largely topographically ordered [12] and thus, small differences in size ratios can be neglected.

On the other hand, the exceptional case of the striatum requires more analysis, since its enormous size makes it significantly larger, approximately 145 times in rats [29], than the other BG structures. Therefore, in this simulation the striatum is modelled using only 600 D1-like and 600 D2like MSNs (FSIs are neglected due to their small number) while STN, GPe and GPi/SNr comprise 150 neurons each.

Finally, lateral inhibition within the striatum has also been neglected here as it is believed to be strong only locally [30] and even this effect has been repeatedly challenged [15], [31].

Our model implies that the $N$ simulated channels encode the same type of information that corresponds to competing actions or behaviours. It is unknown, however, to what extent these representations should be spatially located close enough to be under the influence of lateral inhibition, and this is a feature that requires further investigation.

\section{B. Neural dynamics}

The model that was employed to simulate the membrane potential of individual neurons is the phenomenological "simple model" proposed by Izhikevich [32], [33].

$$
\begin{gathered}
C \frac{d v}{d t}=k\left(v-v_{r}\right)\left(v-v_{t}\right)-u+I+C \mathcal{N}\left(0, \sigma^{2}\right) \\
\frac{d u}{d t}=a\left(b\left(v-v_{r}\right)-u\right)
\end{gathered}
$$

where $v$ is the membrane potential, $u$ a phenomenological recovery variable, $I$ the dendritic and synaptic current, $C$ the membrane capacitance, $v_{r}$ the resting membrane potential, $v_{t}$ the instantaneous threshold potential and finally $a, b$ and $k$ are abstract parameters of the model. The neuron fires a spike when the voltage exceeds the threshold value $v_{\text {peak }}$ and the variables of the model reset to

$$
\begin{aligned}
& v \rightarrow c \\
& u \rightarrow u+d
\end{aligned}
$$

where $c$ and $d$ are further abstract parameters.

With the correct tuning of its parameters, this model is able to exhibit all known types of dynamical behaviour in cortical cells, and to quantitatively reproduce their sub-threshold, spiking, and bursting activity in response to pulses of DC current [33].

Although this model is shown to be equivalent to a simpler form [32], which reduces the number of independent parameters to four, we chose to use equations 1 and 2 because in this form, the majority of the parameters and the variables acquire biophysical meaning. For instance, the membrane potential is represented in $\mathrm{mVolts}$ and the current in $p$ Amperes.

In addition, the term $\mathcal{N}\left(0, \sigma^{2}\right)$ of equation 1 represents a general Gaussian noise factor that is added to the membrane potential of each neuron in every simulation step with a constant standard deviation of $\sigma=0.3 \mathrm{mV}$.

Finally, in order to achieve a high level of heterogeneity in the network, a degree of stochastic perturbation was applied to the capacitance $C$ of each neuron sampled from a Gaussian distribution with mean $C_{\mu}$ and standard deviation $0.1 \times C_{\mu}$.

\section{Synaptic dynamics}

To determine the basic chemical synaptic input to the simulated neurons, we followed a standard conductancebased approach [34]. Each synapse that connects the presynaptic neuron $i$ to the post-synaptic neuron $j$ is modelled as

$S_{i j}^{x}(t)= \begin{cases}g_{i j} e^{-\left(t-\left(t_{i}+\lambda\right)\right) / \tau_{x}}\left(E_{x}-v_{j}\right) & \text { if } t \geq\left(t_{i}+\lambda\right) \\ 0 & \text { if } t<\left(t_{i}+\lambda\right)\end{cases}$

where $t$ is the current simulation time, $t_{i}$ is the time of last firing of neuron $i, x$ is the type of the synaptic receptor, $\lambda$ is the delay of the synapse, $g_{i j}$ is the maximum conductance of the synapse, i.e. the weight of the underlying connection, $E_{x}$ is the synaptic reversal potential and $\tau_{x}$ is the synaptic decay time constant. When the effect of a new spike reaches the post-synaptic neuron $j, S_{i j}$ jumps to the value $g_{i j}$ and decays exponentially with rate $\tau$.

Different values of $\tau$ denote different pairings of neurotransmitter and synaptic receptor and represent the duration of a neurotransmitter re-uptake and dispersal. The neurotransmitter that is thought to be dominant in the excitatory

\footnotetext{
${ }^{1}$ Transformation of the parameters found in [36], for $R=18$ MOhms, $\tau=6 \mathrm{~ms}$ and $v_{\text {peak }}=20 \mathrm{~ms}$ (the latter parameters were taken from [4])
} 
TABLE I

IZHIKEVICH NEURON PARAMETERS OF THE BASAL GANGLIA NUCLEI.

\begin{tabular}{|c|c|c|}
\hline \multicolumn{3}{|c|}{ STRIATUM D1,D2 } \\
\hline$a$ & 0.01 & Taken from [35] \\
\hline$b$ & -20 & -" - \\
\hline$c$ & $-55 m V$ & -" - \\
\hline$d$ & 91 & -" - \\
\hline$v_{r}$ & $-80 m V$ & -" - \\
\hline$v_{t}$ & $-29.7 m V$ & -"- \\
\hline$v_{\text {peak }}$ & $40 m V$ & -"- \\
\hline$C_{\mu}$ & $15.2 p F$ & -" - \\
\hline$k$ & 1 & - \\
\hline$d_{1}$ & $30 \%$ & Taken from [4], (in D1) \\
\hline$d_{2}$ & $30 \%$ & Taken from [4], (in D2) \\
\hline \multicolumn{3}{|c|}{ STN } \\
\hline$a$ & 0.005 & Derived from $[36]^{1}$ \\
\hline$b$ & 88.33 & - \\
\hline$c$ & $-65.0 m V$ & -" - \\
\hline$d$ & 500.0 & -"- \\
\hline$v_{r}$ & $-61.0 m V$ & -"- \\
\hline$v_{t}$ & $-64.035 \mathrm{mV}$ & -"- \\
\hline$v_{\text {peak }}$ & $20.0 \mathrm{mV}$ & Taken from [4] \\
\hline$C_{\mu}$ & $333.33 p F$ & Derived from $[36]^{1}$ \\
\hline$k$ & 13.33 & -" - \\
\hline$d_{2}$ & $30 \%$ & Taken from [4] \\
\hline \multicolumn{3}{|c|}{ GPe } \\
\hline$a$ & 0.05 & Derived from [7] \\
\hline$b$ & 2.5 & -"- \\
\hline$c$ & $-60 m V$ & -"- \\
\hline$d$ & 70 & -"- \\
\hline$v_{r}$ & $-55.1 m V$ & -" - \\
\hline$v_{t}$ & $-54.7 m V$ & -"- \\
\hline$v_{\text {peak }}$ & $15 \mathrm{mV}$ & -"- \\
\hline$C_{\mu}$ & $40 p F$ & -"- \\
\hline$k$ & 0.706 & -" - \\
\hline$d_{2}$ & $30 \%$ & Taken from [4] \\
\hline \multicolumn{3}{|c|}{ GPi/SNr } \\
\hline$a$ & 0.05 & Derived from [7] \\
\hline$b$ & 3 & -" - \\
\hline$c$ & $-65 m V$ & -"- \\
\hline$d$ & 200 & -"- \\
\hline$v_{r}$ & $-55.8 m V$ & -"- \\
\hline$v_{t}$ & $-55.2 m V$ & -"- \\
\hline$v_{\text {peak }}$ & $20.0 \mathrm{mV}$ & -"- \\
\hline$C_{\mu}$ & $80 p F$ & -"- \\
\hline$k$ & 1.731 & -" - \\
\hline
\end{tabular}

synapses of this simulation is glutamine and the corresponding receptors in the postsynaptic neurons are $A M P A$ and NMDA. Also, all the inhibitory neurons are gabaergic i.e. the corresponding neurotransmitter is $\gamma$-Aminobutyric acid, which binds to $G A B A_{A}$ receptors.

The parameters that have been used to model the different categories of synapses are given in table III.

Hence, the synaptic input current $I$ of the equation 1 that the neuron $j$ receives over time is described as

$$
I_{j}=\sum_{i, x} S_{i j}^{x}+I_{\text {spon }}
$$

The first term of this equation represents the total synaptic current $I_{s y n}=I_{s y n}^{a m p a}+I_{s y n}^{n m d a}+I_{s y n}^{g a b a}$ and the parameter $I_{\text {spon }}$ represents a constant depolarizing current that, after calibration, allows the model to show spontaneous activity of the corresponding cells, similar to empirical data. Humphries et al in [4] have tuned this parameter to match the firing rates of the $\mathrm{BG}$ nuclei to empirical data of awake resting
TABLE II

SYNAPTIC MODEL WITH DOPAMINE [4].

$$
\begin{array}{ll}
\text { Striatum D1 } & \left(I_{\text {syn }}^{a m p a}+I_{s y n}^{n m d a}\right)\left(1+d_{1}\right) \\
\text { Striatum D2 } & \left(I_{s y n}^{a m p a}+I_{s y n}^{n m d a}\right)\left(1-d_{2}\right) \\
\text { STN } & \left(I_{s y n}^{a m p a}+I_{s y n}^{n m d a}\right)\left(1-\alpha_{1} d_{2}\right)+I_{\text {syn }}^{\text {gaba }}\left(1-\alpha_{2} d_{2}\right)+I_{\text {spon }} \\
& I_{\text {spon }}=11 \times 10^{-10} \mathrm{Amp} \\
& \alpha_{1,2}=0.5 \\
& \left(I_{\text {syn }}^{a m p a}+I_{\text {syn }}^{n m d a}\right)\left(1-\beta_{1} d_{2}\right)+I_{\text {syn }}^{\text {gaba }}\left(1-\beta_{2} d_{2}\right)+I_{\text {spon }} \\
& I_{\text {spon }}=3.8 \times 10^{-10} \mathrm{Amp} \\
\text { GPe } & \beta_{1,2}=0.5 \\
& I_{\text {syn }}^{\text {ampa }}+I_{\text {syn }}^{\text {nmda }}+I_{\text {syn }}^{\text {gaba }}+I_{\text {spon }} \\
& I_{\text {spon }}=3.9 \times 10^{-10} \mathrm{Amp} \\
\text { GPi/SNr } &
\end{array}
$$

\begin{tabular}{|c|c|}
\hline \multicolumn{2}{|c|}{ GENERAL } \\
\hline$p_{\text {con }}$ & 0.25 \\
\hline$g_{i j}$ & $1 n S$ \\
\hline$\lambda_{\text {Cortex }-S D 1 / 2}$ & $10 \mathrm{~ms}$ \\
\hline$\lambda_{\text {Cortex }-S T N}$ & $2.5 \mathrm{~ms}$ \\
\hline$\lambda_{S T N-S N r}$ & $1.5 \mathrm{~ms}$ \\
\hline$\lambda_{S T N-G P e}$ & $2 m s$ \\
\hline$\lambda_{S D 1-S N r}$ & $4 \mathrm{~ms}$ \\
\hline$\lambda_{S D 2-G P e}$ & $5 \mathrm{~ms}$ \\
\hline$\lambda_{G P e-S T N}$ & $4 m s$ \\
\hline$\lambda_{G P e-S N r}$ & $3 \mathrm{~ms}$ \\
\hline$\lambda_{G P e}$ & $1 \mathrm{~ms}$ \\
\hline$\lambda_{S N r}$ & $1 \mathrm{~ms}$ \\
\hline \multicolumn{2}{|c|}{ EXCITATORY } \\
\hline$E_{a m p a}$ & $0 m V$ \\
\hline$E_{n m d a}$ & $0 m V$ \\
\hline$\tau_{a m p a}$ & $2 \mathrm{~ms}$ \\
\hline$\tau_{n m d a}$ & $100 \mathrm{~ms}$ \\
\hline \multicolumn{2}{|c|}{ INHIBITORY } \\
\hline$E_{g a b a}$ & $-80 m V$ \\
\hline$\tau_{g a b a}$ & $3 \mathrm{~ms}$ \\
\hline
\end{tabular}

TABLE III

SYNAPTIC PARAMETERS (TAKEN FROM [4]).

rats. Although we used a different neuron model, crucial physiological parameters such as the spiking thresholds $v_{\text {peak }}$ have been largely maintained and our model produced similar firing rates. Hence, the values of the original model were kept and they are shown in table II.

Additionally, the majority of the synapses in the network are under the influence of dopamine, which either facilitates or suppresses the input of the neuron, depending on the dopamine receptor type that this neuron expresses. The method to model the effects of dopamine in each BG nuclei is a simplified adaptation from Humphries et al [4]. The level of dopamine in the system is reflected in $d_{1} / d_{2}$ for $D 1 / D 2$ - type receptors respectively, and under normal conditions $d_{1}=d_{2}$. Hence, the final input $I$ of each type of neuron in equation 1 takes the form that is also shown in table II. 


\section{Cortical stimulation}

The main input to the $\mathrm{BG}$ is glutamatergic and comes from afferent axons of pyramidal neurons in layer $\mathrm{V}$ of different areas of the cortex as well as the thalamus. Despite this diversity, the BG consist of the same repeating internal circuitry [12] which is also largely retained in most vertebrate species [37]. This suggests that their function is very similar and independent of what their input stands for. Following this rationale, this study examines the oscillatory nature of the input signals, rather than lower-level characteristics that vary across cortical regions.

Hence, the stimulation of the BG model is realised through a number $\left(N_{i}\right)$ of inhomogeneous Poisson processes with rate parameter

$$
\lambda_{i}(t)=A_{i} \cos \left(2 \pi f_{i} t+\varphi_{i}\right)
$$

where $A_{i}$ is the amplitude, $f_{i}$ the frequency, and $\varphi_{i} \in$ $[0,2 \pi)$ the phase of the oscillatory input $T_{i}$.

Similarly to previous models [2], [4] that also rely on the action selection hypothesis, the amplitude of $T_{i}$, also reflected in the firing rate of striatal neurons, represents the salience of the corresponding input [1].

In this study, two competing oscillatory sources $T_{1}$ and $T_{2}$ have been used, with size $N_{1,2}=1000, \varphi_{1}=0$ and thus $\varphi_{2}$ representing the phase offset $\varphi_{\text {off }}=\varphi_{2}-\varphi_{1}$ between $T_{1}$ and $T_{2}$. The amplitude of $T_{1}$ is set to be $A_{1}=30$ spikes/s while the second input is more salient with $A_{2}=60$ spikes/s. Although we used only two inputs, a third channel was simulated in order to observe the behaviour of adjacent inactive BG areas as well as to maintain consistency with [4].

\section{E. Effectiveness metric}

To measure the effectiveness of the model as an action selection mechanism, we define the metric $\epsilon$ that shows how well the BG are able to select one out of a number of distinctive parallel active channels. A good indicator of this performance is the firing activity of the BG's main output nucleus, the SNr-GPi complex, which provide tonic inhibition to the thalamus and brainstem. The action selection hypothesis suggests that by stopping this inhibition in a specific area of the thalamus, the BG let information flow back to the cortex motor areas and thus select the corresponding microscopic channel [38], [1].

As mentioned before, this study relies on the assumption that the firing rate of each channel at the level of the striatum encodes the salience of the represented action. Therefore, it is always straightforward to define which channel should be selected each time by an ideal action selection mechanism.

Taking this into account, the first term of the metric $\epsilon$ is defined as the average ratio of spike events in the active channels of the SNr-GPi complex that should not be selected, against the correct active channel. That is, if channel $A$ is the channel with the highest salience for each point of time, $B$ and $C$ the competitor channels, and $S P_{X}$ the number of spikes in channel $X$ then

$$
a=\frac{S P_{B}+S P_{C}}{S P_{A}+S P_{B}+S P_{C}}
$$

Furthermore, it is important that inhibition of the remaining channels occurs independently of the intensity of the cortical input, in order to reduce the possibility of a false selection. Thus, to secure that the inhibition in channels $B$ and $C$ follows a similar pattern, the second term of the metric $\epsilon$ is given as

$$
b=\frac{2 \cdot \min \left\{S P_{B}, S P_{C}\right\}}{S P_{B}+S P_{C}}
$$

Hence, the complete metric takes the following form.

$$
\epsilon=\left(100 \cdot a^{4} \cdot b\right) \%
$$

The term $a$ in equation 9 is raised to the power of 4 to emphasise the fact that even a small number of spike events in the selected channel can influence significantly the performance of the mechanism by causing unwanted inhibition.

\section{F. Numerics}

The source code for this simulation was initially written in the Python library Brian [39] and then reproduced in $\mathrm{C}++$, using NeMo spiking neural network simulator [40] to achieve a massive parallelization using CUDA. The numerical integration of the model in Python was carried out using the Euler method with an integration time step of $d t=0.25 \mathrm{~ms}$, while the fourth-order Runge-Kutta method with the same time step was later used in $\mathrm{C}++$. Both methods produced similar results.

\section{RESULTS}

Following the methodology described in section III, we conducted a series of simulations that varied the phase offset $\varphi_{\text {off }}$, the frequency of the oscillatory inputs $f_{1,2}=f_{1}=f_{2}$ and the level of dopamine $d_{1,2}=d_{1}=d_{2}$, to identify the critical conditions that affect the efficiency $\epsilon$ of the selection mechanism of the BG. An overview of the results is shown in Figure 2.

Based on this figure, we can make a number of testable predictions about the operation of the BG. First, our results clearly indicate that the critical areas that can affect the behaviour of the BG are all located in low frequencies while gamma oscillations have no discernible effect. Second, highbeta frequencies are able to facilitate selection significantly, but only in the case that the input signals are coherent and a strong signal precedes in time a second, weaker signal with a small phase offset. Third, the frequency range of this advantage is inversely proportional to the level of dopamine in the system. Fourth, the system is ineffective at frequencies lower than $20 \mathrm{~Hz}$ and the phase offset, again, plays a significant role, mainly in low-beta and theta bands.

Furthermore, our results indicate another frequencydependent effect of dopamine. As shown in Figure 2.B and $\mathrm{C}$, when the input signals oscillate at frequencies between 20 and $30 \mathrm{~Hz}$, increased levels of dopamine suppress the effect that is caused by coherent phase-offset on BG selectivity. On the other hand, in the first critical area of theta oscillations 

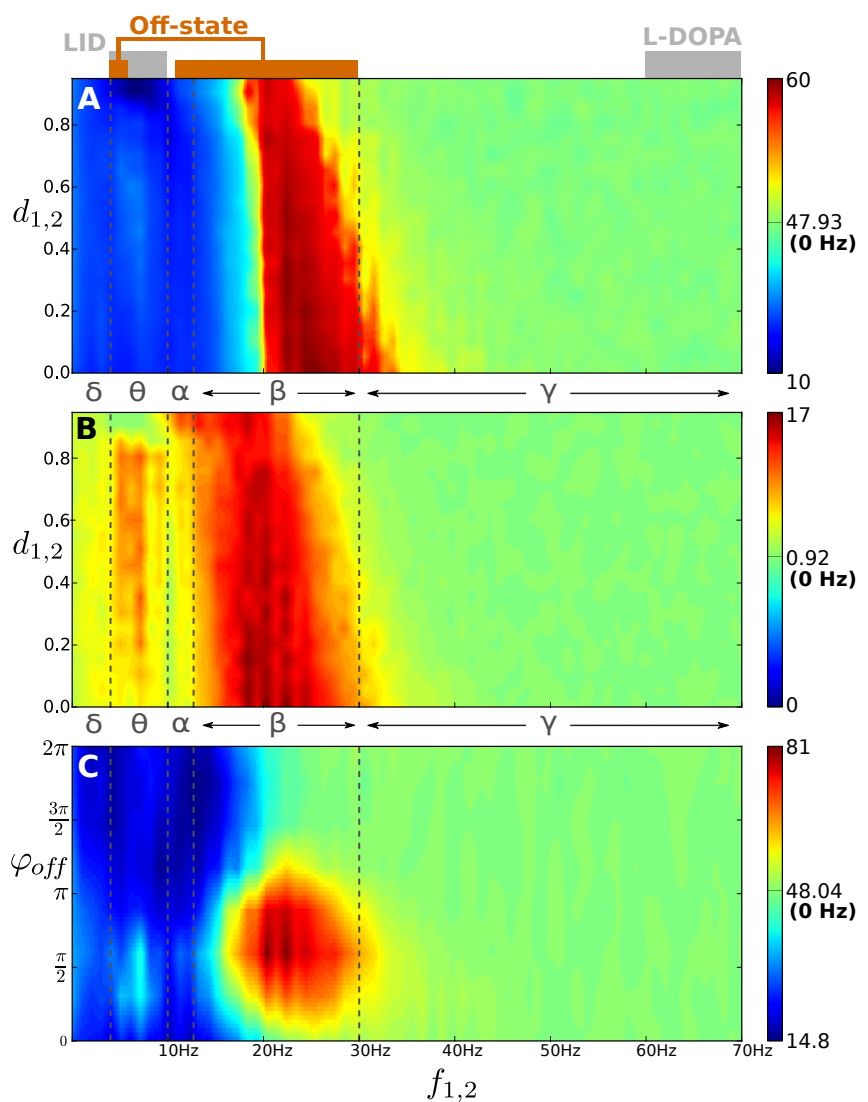

Fig. 2. Effectiveness $\epsilon$ of the model for each combination of dopamine levels $d_{1,2}$ and input frequencies $f_{1,2}$. The colour bars represent the mean (A) and the standard deviation (B) of a sample of 160 simulations with random phase offsets $\varphi_{\text {off }} \in[0,2 \pi)$. C: Mean effectiveness for $d_{1,2}=0.3$ and different phase offsets $\varphi_{\text {off } f}$. In all cases, the green baseline represents $\epsilon$ under normal conditions and no oscillation, i.e. $d_{1,2}=0.3, f_{1,2}=0 \mathrm{~Hz}$.

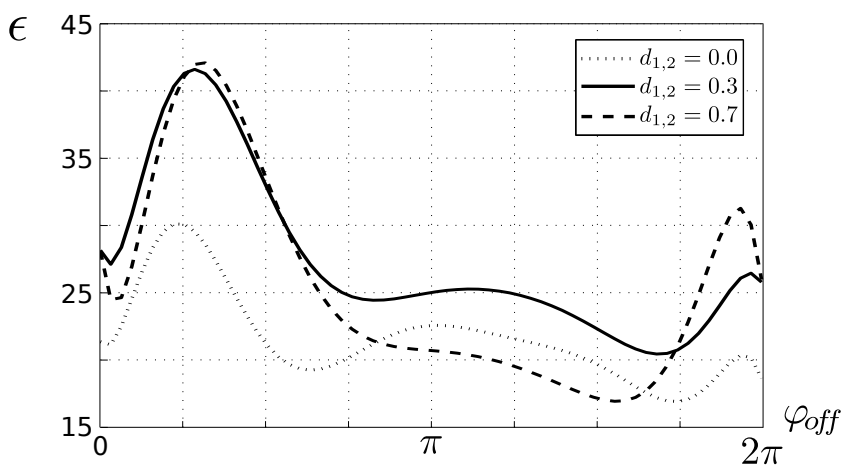

Fig. 3. $5^{\text {th }}$ degree polynomial regression of data that show $\epsilon$ for different phase offsets and $f_{1,2}=7 \mathrm{~Hz}$. This curve was fitted for illustration purposes from a sample of 640 simulations.

$(5-10 \mathrm{~Hz})$, dopamine is clearly proportional to the existence of this effect (Figure 3).

Our model's behaviour in low frequencies is in line with the intuition that the BG operate as a classical winner-takesall (WTA) mechanism. In this context, the two input signals $T_{1}$ and $T_{2}$ can be considered the "competitors" while the cycle of a slow oscillation represents a competition round. A small phase offset $\varphi_{\text {off }}$ can be then interpreted as a temporal advantage for $T_{2}$ over $T_{1}$. Indeed, the effectiveness of the model was significantly stronger in the case of such a temporal advantage for the most salient input $T_{2}\left(\varphi_{\text {off }}<\pi\right.$ in Figure 2.C). In addition, it is worth noting that small phase offsets were proven to be more advantageous than $\varphi_{\text {off }}=0$, when the two inputs are completely synchronized.
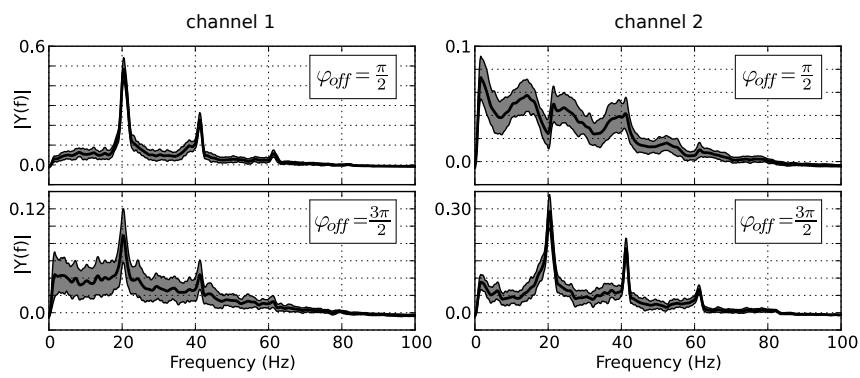

Fig. 4. Frequency spectrum of the SNr-GPi complex when both $\mathrm{T} 1$ and T2 oscillate at $f_{1,2}=20 \mathrm{~Hz}$. The lines represent the mean value and the gray area the SD of a sample of 100 simulations.

The variation of the $\mathrm{BG}$ effectiveness is also reflected in the coherence between the cortical input and the $\mathrm{SNr}$ GPi complex, the output of the BG model. In Figure 4, a Fourier transform of the binned spiking activity of SNr-GPi, in 100 simulations with $f_{1,2}=20 \mathrm{~Hz}$, shows that although the frequency of the input signal is generally maintained through the BG structures, its intensity varies largely between the BG's microscopic channels, depending on the channel that is currently selected. In the first case, when $\varphi_{o f f}=\frac{\pi}{2}$, the system is very effective and clearly selects the most salient input, in this case channel 2. For $\varphi_{o f f}=\frac{3 \pi}{2}$, the lesssalient channel 1 is selected, though both active channels are entrained at the input frequency.

\section{DISCUSSION}

The neural model that is presented in this paper, uses "Izhikevich" neurons with particular sets of parameters that make them able to exhibit the dynamical behaviour found in the real neurons of the BG nuclei. In initial experiments, we also tried different less realistic models with simple eventbased synapses, more general parameters for the neuron equations as well as different integration methods (Euler and Runge-Kutta methods). However, these simulations produced similar results and always indicated that, in low bands, a small phase offset gives a clear advantage to the strongest input signal and thus influences competition in the BG.

Revisiting the current literature, a number of studies show interesting relations with our results.

The first important relation can be found in studies of human patients with Parkinson's disease that receive pharmacological treatment. Local field potential recordings in STN [41], [42] and GPi [42] as well as single neuron recordings [17] have shown that when the patients are in the "off" medication (Parkinsonian) state, oscillatory activity in 
the aforementioned areas exhibits two peaks, one in the beta band $(11-30 \mathrm{~Hz})$ in all patients and one in the low-theta band $(4-6 \mathrm{~Hz})$ in patients with tremor. However, after the administration of the biochemical L-DOPA (or levodopa), the balance of the oscillations in the BG changes dramatically. In the above studies, patients showed a major reduction in activity within the two bands that prevailed before and a significant increment in the gamma band $(>60 \mathrm{~Hz})$ [41]. Furthermore, patients that developed levodopa-induced dyskinesia (LID), a side-effect after chronic administration of L-DOPA, showed a second rather higher increment in the hippocampal theta frequencies $(4-10 \mathrm{~Hz})$ [41].

Interestingly, the results that are depicted in Figure 2 also make a clear distinction between the above frequency bands and suggest that, for robust BG performance, cortical inputs have to either be oscillating in high-frequency bands, as in the case with L-DOPA, or be coherent in a particular manner.

Specifically, our interpretation of low-frequency oscillation cycles defining short "competition rounds" in the BG's action selection mechanism provides a possible explanation of the fact that Parkinsonian resting tremor, a very common symptom of PD, is mainly associated with theta (and not beta [43]) BG activity [44], [17], [19]. Our simulations indicate that the timing of a single competition round should be between $\frac{1}{15}$ and $\frac{1}{30}$ seconds because the $15-30 \mathrm{~Hz}$ band is the critical area where selectivity is enhanced (Figure 2.C). Hence, at theta frequencies, an oscillation cycle can accommodate more than one competition round and it is likely that two different competing signals with a large phase offset will be able to win in different rounds.

In addition, in [28], data from rats on a Y maze showed that strong theta coherence between the PFC and the hippocampus, two major inputs of the $\mathrm{BG}$, peaks at the choice point of a learnt rule, causing entrainment of the PFC activity to hippocampal theta oscillations. Indeed, administration of dopamine in the PFC was found to cause the same effects and increase theta coherence between these two structures. This led to the hypothesis that the intensity of theta coherence, at this level, represents reward expectation. In our simulations, we found critical effects in the selectivity of competing theta-coherent inputs in our BG model. These effects are modulated by dopamine, which promotes the variation of the BG efficiency, depending on the phase offset $\varphi_{\text {off }}$ (Figure 3). This is compatible with the above theory indicating that dopamine increases the necessity for theta phase-locking (and thus the necessity for coherence) at the level of the BG input.

The above two interpretations of the role of theta rhythms do not contradict each other since, for the existence of resting tremor, a large phase offset is assumed, while for $\varphi_{\text {off }} \simeq \frac{\pi}{4}$, the $\mathrm{BG}$ effectiveness is shown to be close to the baseline (Figure 3). On the contrary, it is possible that in case that theta activity is required at the level of the BG inputs, maybe due to a memory-related or other cognitive process, an increase of dopamine filters the non-coherent input signals, while it also promotes theta coherence, achieved by phase shifts of the PFC neurons [28].
Furthermore, our results are also consistent with the modern view that beta activity at the level of the $\mathrm{BG}$, is inversely proportional to the likelihood of a new goal-oriented movement [25]. This hypothesis also states that the dopaminergic system modulates the level of beta cortical entrainment of the $\mathrm{BG}$ and, in tonic rates, it maintains BG beta activity in low levels. According to our model, exaggerated beta activity increases the advantage of a salient action over a less-salient alternative, under particular conditions of their phase offset. This relation with saliency suggests that when the BG are entrained to cortical beta rhythms, potential attempts for new voluntary actions, that have not yet been associated with reward, will be suppressed.

Finally, dopamine was found to have a complementary role to the BG oscillatory mechanisms. Rather than only preventing beta synchronization [25], it was also shown to suppress the effects of beta oscillations on the BG circuitry (Figure 2.A and B).

\section{CONCLUSIONS}

This paper has described a neural model of the standard BG circuitry which, to our knowledge, is the first that is both complete and based on Izhikevich's simple model of spiking neurons [32]. In addition, an analysis of the relationship between frequency in cortical oscillations, dopamine and the effectiveness of the BG as an action selection device revealed an explanation of the importance of phase-locked oscillations in low bands. However, how oscillations in these bands are modulated or phase-locked in healthy cortical structures, still remains an open question.

During our investigation, we made three fundamental assumptions. First, we aligned with the premise that the BG operates as a central action selection unit that is able to resolve potential conflicts between motor or cognitive resources [1], [2]. Second, we assumed that microscopic channels of parallel isolated loops within the BG represent different resources, and that the activation of each channel at the level of the striatum encodes the salience of the corresponding action [1], [4]. Third, we assumed that cortical signals that reach the $\mathrm{BG}$ oscillate at frequencies between 0.1 and $100 \mathrm{~Hz}$.

We are currently extending our model to investigate how the short- and long-term effects of plasticity influence the BG selectivity or the critical frequencies in Figure 2. Finally, we are planning to conduct further analysis based on more meaningful cortical input, where coalitions of cortical oscillators have more complex frequency spectrum and cooperate in order to win the BG competition.

\section{ACKNOWLEDGEMENTS}

The authors would like to thank Dr Mark Humphries for the valuable advices and discussions. This work was supported by an EPSRC doctoral training grant.

\section{REFERENCES}

[1] P. Redgrave, T.J. Prescott and Kevin Gurney, "The basal ganglia: a vertebrate solution to the selection problem?," Neuroscience, vol. 89, no. 4, pp. 1009-1023, 1999. 
[2] K. Gurney, T.J. Prescott and P. Redgrave, "A computational model of action selection in the basal ganglia. I. A new functional anatomy," Biological Cybernetics, vol. 84, no. 6, pp. 401-410, 2001.

[3] A.J.N. Holgado, J.R. Terry and R. Bogacz, "Conditions for the generation of beta oscillations in the subthalamic nucleus-globus pallidus network", The Journal of Neuroscience, vol. 30, no. 37, pp. 12340$12352,2010$.

[4] M.D. Humphries, R.D. Stewart and K.N. Gurney, "A physiologically plausible model of action selection and oscillatory activity in the basal ganglia," The Journal of Neuroscience, vol. 26, no. 50, pp. 1292112942,2006

[5] A. Kumar, S. Cardanobile, S. Rotter and A. Aertsen, "The role of inhibition in generating and controlling Parkinsons disease oscillations in the Basal Ganglia", Frontiers in Systems Neuroscience, vol. 5, no. 86, 2011.

[6] T.C. Stewart, T. Bekolay and C. Eliasmith, "Learning to select actions with spiking neurons in the Basal Ganglia", Frontiers in Neuroscience, vol. 6, no. 2, 2012.

[7] M. Lindahl, I.K. Sarvestani, O. Ekeberg and J.H. Kotaleski, "Signal enhancement in the output stage of the basal ganglia by synaptic short-term plasticity in the direct, indirect, and hyperdirect pathways", Frontiers in Computational Neuroscience, vol. 7, no. 76, 2013.

[8] T.J. Prescott, F.M. Montes González, K. Gurney, M.D. Humphries and P. Redgrave, "A robot model of the basal ganglia: behavior and intrinsic processing", Neural Networks, vol. 19, no. 1, pp. 31-61, 2006.

[9] C. Eliasmith, T.C. Stewart, X. Choo, T. Bekolay, T. DeWolf, C. Tang and D. Rasmussen, "A large-scale model of the functioning brain", Science, vol. 338, no. 6111, pp. 1202-1205, 2012.

[10] M.D. Bevan, P.J. Magill, D. Terman, J.P. Bolam and C.J. Wilson, "Move to the rhythm: oscillations in the subthalamic nucleus-external globus pallidus network.", Trends in Neurosciences, vol. 25, no. 10, pp. 525-531, 2002.

[11] J.D. Berke, M. Okatan, J. Skurski and H.B. Eichenbaum, "Oscillatory entrainment of striatal neurons in freely moving rats", Neuron, vol. 43, no. 6, pp. 883-896, 2004.

[12] G.E. Alexander, M.R. DeLong and P.L. Strick, "Parallel organization of functionally segregated circuits linking basal ganglia and cortex", Annual Review of Neuroscience, vol. 9, no. 1, pp. 357-381, 1986.

[13] A. Nambu, "Seven problems on the basal ganglia", Current Opinion in Neurobiology, vol. 18 no. 6, pp. 595-604, 2008.

[14] J.P. Bolam, H. Bergman, A.M. Graybiel, M. Kimura, Plenz D, H.S. Seung, D.J. Surmeier and J.R. Wickens, "Microcircuits in the striatum", In: S. Grillner, A.M. Graybiel, eds. Microcircuits: The Interface Between Neurons and Global Brain Function. Cambridge MA: MIT Press, pp. 165-190, 2006.

[15] M.D. Humphries, R. Wood, and K. Gurney, "Reconstructing the three-dimensional GABAergic microcircuit of the striatum", PLoS Computational Biology, vol. 6, no. 11, pp. e1001011, 2010.

[16] R.L. Albin, A.B. Young and J.B. Penney, "The functional anatomy of basal ganglia disorders", Trends in Neurosciences, vol. 12, no. 10, pp. 366-375, 1989

[17] R. Levy, W.D. Hutchison, A.M. Lozano and J.O. Dostrovsky, "Highfrequency synchronization of neuronal activity in the subthalamic nucleus of parkinsonian patients with limb tremor", The Journal of Neuroscience, vol. 20, no. 20, pp. 7766-7775, 2000.

[18] A.G. Androulidakis, C. Brucke, F. Kempf, A. Kupsch, T. Aziz, K. Ashkan, A.A. Kuhn and P. Brown, "Amplitude modulation of oscillatory activity in the subthalamic nucleus during movement", European Journal of Neuroscience, vol. 27, no. 5, pp. 1277-1284, 2008.

[19] P. Tass, D. Smirnov, A. Karavaev, U. Barnikol, T. Barnikol, I. Adamchic, C. Hauptmann, N. Pawelcyzk, M. Maarouf, V. Sturm, H.J. Freund and B. Bezruchko, "The causal relationship between subcortical local field potential oscillations and Parkinsonian resting tremor", Journal of Neural Engineering, vol. 7, no. 1, 2010.

[20] S. Little, A. Pogosyan, A.A. Kuhn and P. Brown, "Beta band stability over time correlates with Parkinsonian rigidity and bradykinesia", Experimental Neurology, vol. 236, no. 2, pp. 383-388, 2012.

[21] A.M. Lozano and N. Lipsman, "Probing and regulating dysfunctional circuits using deep brain stimulation", Neuron, vol. 77, no. 3, pp. 406-424, 2013.

[22] P. Brown, A. Kupsch, P.J. Magill, A. Sharott, D. Harnack and W. Meissner, "Oscillatory local field potentials recorded from the subthalamic nucleus of the alert rat", Experimental Neurology, vol. 177, no. 2, pp. 581-585, 2002.
[23] A.K. Engel and P. Fries, "Beta-band oscillations-signalling the status quo?", Current Opinion in Neurobiology, vol. 20, no. 2, pp. 156-165, 2010.

[24] E. Stein and I. Bar-Gad, "Beta oscillations in the cortico-basal ganglia loop during parkinsonism", Experimental Neurology, vol. 245, pp. 5259, 2013.

[25] N. Jenkinson and P. Brown, "New insights into the relationship between dopamine, beta oscillations and motor function", Trends in Neurosciences, vol. 34, no. 12, pp. 611-618, 2011.

[26] J.A. Obeso, M.C. Rodriguez-Oroz, B. Benitez-Temino, F.J. Blesa, Jorge Guridi, C. Marin and M Rodriguez, "Functional organization of the basal ganglia: therapeutic implications for Parkinson's disease", Movement Disorders vol. 23, no. S3, pp. S548-S559, 2008.

[27] V. Litvak, A. Jha, A. Eusebio, R. Oostenveld, T. Foltynie, P. Limousin, L. Zrinzo, M.I. Hariz, K. Friston and P. Brown, "Resting oscillatory cortico-subthalamic connectivity in patients with Parkinsons disease", Brain, vol. 134, no. 2, pp. 359-374, 2011.

[28] K. Benchenane, A. Peyrache, M. Khamassi, P.L. Tierney, Y. Gioanni, F.P. Battaglia, and S.I. Wiener, "Coherent Theta Oscillations and Reorganization of Spike Timing in the Hippocampal- Prefrontal Network upon Learning”, Neuron vol. 66, no. 6, pp. 9210-36, 2010.

[29] D.E. Oorschot, "Total number of neurons in the neostriatal, pallidal, subthalamic, and substantia nigral nuclei of the rat basal ganglia: a stereological study using the cavalieri and optical disector methods", The Journal of Comparative Neurology, vol. 366, no. 4, pp. 580-99, 1996.

[30] J. Wickens, "Basal ganglia: structure and computations", Network: Computation in Neural Systems, vol. 8, no. 4, pp. R77-R109, 1997.

[31] R. Miller and J. Wickens, "Brain dynamics and the striatal complex", CRC Press, 2003.

[32] E.M. Izhikevich, "Simple model of spiking neurons", IEEE Transactions on Neural Networks, vol. 14, no. 6, pp. 1569-1572, 2003.

[33] E.M. Izhikevich, "Dynamical systems in neuroscience: the geometry of excitability and bursting", MIT press, Cambridge, Massachusetts, 2006.

[34] P. Dayan, L.F. Abbott and L. Abbott, "Theoretical Neuroscience: Computational and Mathematical Modeling of Neural Systems", The MIT Press, Cambridge, Massachusetts, 2001.

[35] M.D. Humphries, N. Lepora, R. Wood and K. Gurney, "Capturing dopaminergic modulation and bimodal membrane behaviour of striatal medium spiny neurons in accurate, reduced models", Frontiers in Computational Neuroscience, vol. 3, no. 26, 2009.

[36] K. Michmizos and K. Nikita, "Local field potential driven Izhikevich model predicts a subthalamic nucleus neuron activity", Annual International Conference of the IEEE Engineering in Medicine and Biology Society, pp. 5900-5903, 2011

[37] A. Reiner, L. Medina and C.L. Veenman, "Structural and functional evolution of the basal ganglia in vertebrates," Brain Research Reviews, vol. 28 , no. 3, pp. 235-285, 1998.

[38] G. Chevalier and J.M. Deniau, "Disinhibition as a basic process in the expression of striatal functions," Trends in Neurosciences, vol. 13, no. 7, pp. 277-280, 1990

[39] D.F. Goodman and R. Brette, "The Brian simulator," Frontiers in Neuroscience, vol. 3, no. 2, pp. 192-197, 2009.

[40] A.K. Fidjeland, and M.P Shanahan, "Accelerated simulation of spiking neural networks using GPUs," IEEE International Joint Conference on Neural Networks, 2010

[41] F. Alonso-Frech, I. Zamarbide, M. Alegre, M.C. Rodriguez-Oroz, J. Guridi, M. Manrique, M. Valencia, J. Artieda and J.A. Obeso, "Slow oscillatory activity and levodopa-induced dyskinesias in Parkinson's disease", Brain, vol. 129, no. 7, pp. 1748-1757, 2006.

[42] P. Brown, A. Oliviero, P. Mazzone, A. Insola, P. Tonali and V. Di Lazzaro, "Dopamine dependency of oscillations between subthalamic nucleus and pallidum in Parkinson's disease", The Journal of Neuroscience, vol. 21, no. 3, pp. 1033-1038, 2001.

[43] A. Kuhn, A. Kupsch, G.H. Schneider and P. Brown, "Reduction in subthalamic $8-35 \mathrm{~Hz}$ oscillatory activity correlates with clinical improvement in Parkinson's disease", European Journal of Neuroscience, vol. 23, no. 7, pp. 1956-1960, 2006.

[44] W.D. Hutchison, A.M. Lozano, R.R. Tasker, A.E. Lang and J.O. Dostrovsky, "Identification and characterization of neurons with tremor-frequency activity in human globus pallidus", Experimental Brain Research, vol. 113, no. 3, pp. 557-563, 1997. 